\title{
Mechanics of Sphincter Action
}

\section{STUDIES ON THE LOWER ESOPHAGEAL SPHINCTER}

\author{
Piero Biancani, Raj K. Goyal, Aris Phillips, and Howard M. Spiro \\ From the Department of Internal Medicine, the Department of Engineering \\ and Applied Science, Yale University, New Haven, Connecticut 06510, and \\ the Department of Medicine, Baylor College of Medicine, Houston, Texas 77025
}

A B S T R A C T The purpose of this study was to evaluate the mechanical factors involved in the genesis of lower esophageal sphincter pressure. We determined the relationship between intraluminal pressure and inside diameter, estimated the ratio between the wall thickness to inside radius, and calculated the tension in the wall of the lower esophageal sphincter as a function of the inside diameter. Various degrees of circumferential stretch' were applied by introducing probes of different diameters in the rat lower esophageal sphincter in vivo. The intraluminal pressure produced by the lower esophageal sphincter around each probe was measured and pressure-diameter curves were constructed during (a) resting state, $(b)$ contraction produced by electrical stimulation, and $(c)$ relaxation produced by esophageal distension. The intraluminal pressure at an inside diameter of $0.5 \mathrm{~mm}$ was similar to that at inside diameter of $3.2 \mathrm{~mm}$. This was true for the sphincter at rest as well as upon electrical stimulation. The pressure diameter curve, however, was sigmoid in shape; at first it showed a decline and then an increase followed by decline in pressure again with increasing diameters. The ratio of wall thickness to inside radius or the magnification factor varied with inside diameters as expected and this ratio increased steeply at small inside diameters. The tension diameter curves of the sphincter muscle showed that optimal tension development occurred not near sphincter closure but at a much wider diameter of $3.2 \mathrm{~mm}$ and that this muscle developed tension even at small luminal diameters. This behavior of the sphincter muscle ensures effective intraluminal pressure over a wide range of luminal diameters.

Dr. Goyal's present address is the Department of Internal Medicine, University of Texas Southwestern Medical School, Dallas, Tex. 75235.

Received for publication 24 June 1971 and in revised form 28 June 1973.

\section{INTRODUCTION}

Manometric studies identify a band of elevated pressure at the lower end of the esophagus $(1,2)$ but the mechanism by which such a high pressure zone is produced is the subject of much speculation. Obviously, any intraluminal pressure in the sphincter area would be generated by the tension developed in the muscle of the sphincter wall. From his studies on blood vessels, Burton $(3,4)$ showed that structures with small inside diameters, such as arterioles and the capillaries, can maintain high intraluminal pressures despite very small tension development in their walls, whereas larger vessels would require much greater tension to hold a comparable pressure. Thus, mechanical factors such as diameter of the lumen and the thickness of the wall may play a very important role in determining the relationship between the wall tension and the intraluminal pressure. The relationship between the wall tension and the intraluminal pressure in the lower esophageal sphincter (LES) ${ }^{1}$ or in any other gastrointestinal sphincter has not hitherto been investigated. We present, here, studies on the LES of the rat to define this relationship.

\section{METHODS}

Studies were performed on 99 Sprague-Dawley-descended rats weighing $200-300 \mathrm{~g}$. The animals were anesthetized with intraperitoneal urethane in the doses of $125 \mathrm{mg} / 100 \mathrm{~g}$ of body weight. The abdomen was opened and the abdominal part of the esophagus was exposed clear of the surrounding structures. A small opening was then made in the upper part of the stomach through which probes were inserted in the LES (Fig. 1). In animals prepared in such a way, regular pull-through technique demonstrates esophageal lower high pressure zone. Esophageal distension produced by injection of small quantities of water in the upper esophagus produces a peristaltic wave in the body

${ }^{1}$ Abbreviations used in this paper: LES, lower esophageal sphincter; OTD, optimal tension development. 


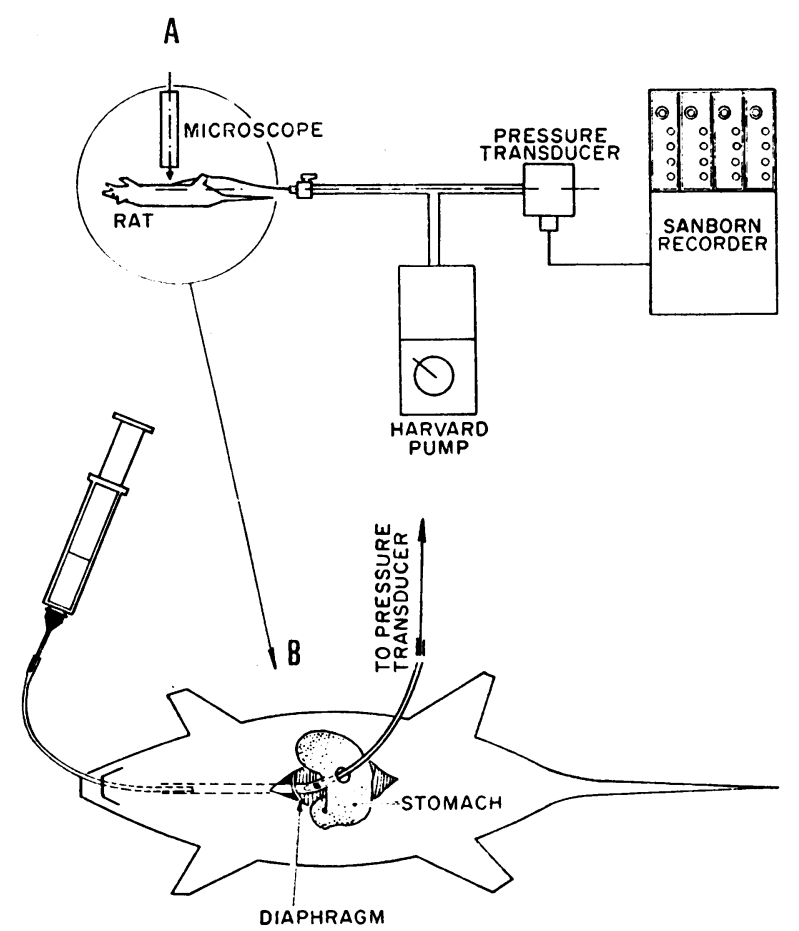

FIgURE 1 Experimental set up. (A) Shows the technique of measurement of outside diameter of the esophagus and the LES. (B) Shows the technique of pressure measurement. Catheters were introduced into the LES and the esophagus through a small opening in the stomach. Distension of upper esophagus was produced by injecting small amounts of water to provoke LES relaxation and peristaltic contraction in the body of the esophagus.

of the esophagus and relaxation of the sphincter similar to that seen in man, dog, and other experimental animals (5).

Six to eight polyvinyl probes of diameter varying from 0.51 to $4.06 \mathrm{~mm}$ (OD) were used. Each probe had a closed tip and a side opening. They were continuously perfused with water by an infusion pump (Harvard Apparatus Co., Inc., Millis, Mass.) and connected to Sanborn pressure transducers (Hewlett-Packard Co., Waltham, Mass.).

The catheters were continuously perfused because in an unperfused catheter, the pressure applied at the catheter tip is not faithfully recorded by the transducer. It has been shown that continuously perfused catheters better quantitate the LES pressure (6); but the steady LES pressure is independent (7) of the rate of infusion. We measured the rat LES pressure at various rates of infusion and found no significant variation: With a catheter of an outside diameter of $2.7 \mathrm{~mm}(I D=1.7 \mathrm{~mm})$ the mean \pm SD LES pressures were $50 \pm 5.6,51.5 \pm 7.7,51.2 \pm 3.0,55 \pm 7.0$, and $52 \pm 2.8 \mathrm{~mm} \mathrm{Hg}$ respectively with perfusion rates of 0.0076 $0.0191,0.0382,0.0764$, and $0.101 \mathrm{~cm}^{3} / \mathrm{min}$. These differences in the pressure were not significant $(P>0.05)$.

In contrast to the steady pressures, the rate of the increase in the recorded pressure $(\Delta P / \Delta t)$ is greatly dependent upon the rate of infusion. The larger rates of infusion will faithfully represent the change in the applied pressure. Conversely, at slower rates of infusion, the peak of pressure may be delayed and truncated. This has been shown to be true for the esophageal contractile wave $(8,9)$. Further, as the deformability of the larger catheters is larger than those of smaller ones, the larger diameter catheters would require a larger flow rate as compared with smaller diameter catheters to faithfully register rapid changes in applied pressure $(10)$.

Therefore, we used small perfusion rates for small probes and higher rates for the larger probes. The rate of perfusion was such that it did not significantly elevate the base-line pressure as a result of viscous resistance. These variations in perfusion rate helped to uniformize the possible variations in the recorded pressure because of the differences in the characteristics of the fluid dynamics in the catheters of different sizes.

Since the LES in the rat is entirely intraabdominal, the position of the catheter could be kept under check in the following way: the site of the opening in the catheter was marked in black and, on illumination of the lower end of the esophagus with high intensity light, the black dot could be easily identified through the semitransparent esophageal wall. The point which gave the highest pressure was chosen for all measurements of the sphincter pressure. The pressure in the body of th eesophagus was measured above the high pressure zone in the intraabdominal esophagus.

The outside diameter of the probe used gave the inside diameter of the sphincter and the body of the esophagus. In this way various known amounts of circumferential stretch were applied. Pressure measurements were made in resting state and during sphincter relaxation produced by esophageal distension. Pressure measurements were also made during electrical stimulation. Square wave pulses of $15 \mathrm{~V}$ at $30 / \mathrm{s}$ frequency with stimulus duration $1 \mathrm{~ms}$ were applied through two silver electrodes using a Grass stimulator (model SD5, Grass Instrument Co., Quincy, Mass.) This produced localized tetanic contraction of the circular as well as the longitudinal muscle of the sphincter and the esophagus. The voltage and frequency of the stimulus was chosen because it produced the maximum increase in the pressure; the pressure did not increase further with increase in the voltage or frequency but reduction in voltage and frequency reduced the response.

Electrical stimulation caused contraction of both muscle layers of the rat esophagus because it has striated muscle in both of its layers. We studied specimens of the gastroesophageal area from 10 rats. As described by Botha (5) both circular and longitudinal layers were composed of striated muscle fibers except at the most distal part of the sphincter area where some smooth muscle fibers appeared in the circular layer. This is different from the behavior of the smooth muscle esophagus of the opossum (11) which upon electrical stimulation shows relaxation of the sphincteric area due to activity of the inhibitory nerves (12). Sustained contraction of rat LES during the period of electrical stimulation can also be demonstrated in vitro (unpublished observations).

Thus, pressure-diameter curves of the LES and the body of the esophagus were constructed in the intact rat at rest, during esophageal peristalsis, and upon electrical stimulation. The contribution of the mucosal folds was not taken into account.

Pressure-diameter curves were also obtained for the esophagus and the LES in the isolated but in situ preparation of the esophagus described previously (13). In this preparation, the rats were anesthetized and the esophagus was exposed after removing the heart, lungs, and other mediastinal structures. The thoracic cavity was used as a 
container. It was filled with constantly circulating Ringer's solution at $37 \pm 1^{\circ} \mathrm{C}$ which was oxygenated by bubbling a mixture of $95 \%$ oxygen and $5 \%$ carbon dioxide through it. In some studies nonoxygenated Ringer's solution was used. The nonoxygenated preparation became nonviable and failed to respond to electrical stimulation.

Average tension in the wall was calculated with the help of Laplaces' Law which defines the relationship of the luminal pressure to the tension in the wall and the radius of the lumen. Burton (3) has shown that this law can be modified for use in thick-walled structures such as blood vessels as follows:

$$
\begin{aligned}
P & =\frac{T \times t}{r} \\
& =T \times t / r \\
& =T \times \mathrm{M} \text { factor, }
\end{aligned}
$$

where $T=$ average tension in the wall; $t=$ thickness of the wall; and $t / r=$ thickness to radius ratio or magnification factor ( $M$ factor).

Thus, to calculate wall tension we needed to know the wall thickness at various diameters. $M$ factor was calculated by dividing the thickness by the radius of the lumen.

The wall thickness was estimated by subtracting the inside diameter, given by the probe introduced into the lumen, from the outside diameter of the sphincter area and dividing by 2 . The outside diameter was directly measured with a measuring microscope (Bausch \& Lomb Inc., Scientific Instrument Div., Rochester, N. Y., model 15) with 20 times magnification, so that $0.05 \mathrm{~mm}$ was magnified to $1 \mathrm{~mm}$. The accuracy of this system was within $0.05 \mathrm{~mm}$.

It is customary to report intraluminal pressures in the esophagus in millimeters of mercury or sometimes in centimeters of water; $1 \mathrm{~mm} \mathrm{Hg}$ being equivalent to $13.6 \mathrm{~mm}$ $\mathrm{H}_{2} \mathrm{O}$. However, tension is usually expressed in units such as gram force per square centimeter $\left(\mathrm{gf} \cdot \mathrm{cm}^{-2}\right) ; 1 \mathrm{gf} \cdot \mathrm{cm}^{-2}$ is equivalent to $1 \mathrm{~cm} \mathrm{H}_{2} \mathrm{O}$ or $0.73 \mathrm{~mm} \mathrm{Hg}$ or $980 \mathrm{dyn} / \mathrm{cm}^{2}$ (14). In this report we will use gram force per square centimeter for both pressure and tension for the sake of convenience and uniformity.

\section{RESULTS}

The pressure record of the sphincter was not a steady straight line. We observed small pressure oscillations, which occurred at a rate of approximately $1 / \mathrm{s}$, that were related to respiratory cycle. The amplitude of these oscillations varied from 1.5 to $4 \mathrm{~mm} \mathrm{Hg}\left(2-5 \mathrm{gf} \cdot \mathrm{cm}^{-2}\right)$. Superimposed on these fast oscillations were irregular slow pressure changes of $10-18 \mathrm{~mm} \mathrm{Hg}$ (15-25 gf. $\left.\mathrm{cm}^{-2}\right)$; their duration was $10-25 \mathrm{~s}$. These slow fluctuations were observed in all the intact animals (Fig. 2).

The steady base-line pressure for each probe was measured for several minutes. Each probe was inserted 3 times and the average of these three numbers was calculated and considered as the pressure for that probe. The largest probe damaged the sphincter in some animals and this was indicated by the inability to reproduce the pressure previously measured with the smaller probe. When this happened, that study was discarded. There

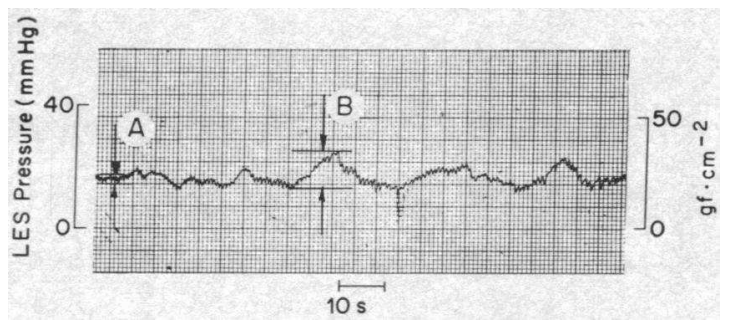

Figure 2 Profile of the LES pressure. The small frequent pressure changes (A) are related to respiration. The large, less frequent pressure elevations (B) occurred spontaneously.

was some variability in the pressures measured at different times. The mean coefficient of variation (i.e., the standard deviation expressed as a percent of the mean value) for the resting LES pressure in the same animal was $13 \%$. The mean coefficient of variation for different animals was $15 \%$.

\section{Pressure-diameter relationship of the LES in intact animals}

Resting state. The mean \pm SD LES pressure in 17 animals was $83 \pm 29 \mathrm{gf} \cdot \mathrm{cm}^{-2}$ at a diameter of $0.51 \mathrm{~mm}$. A comparable pressure of $72 \pm 10 \mathrm{gf} \cdot \mathrm{cm}^{-2}(P>0.5)$ was obtained at a diameter of $3.2 \mathrm{~mm}$. However, significantly lower pressure $(P<0.001)$ was measured at a diameter of $1.63 \mathrm{~mm}$. Full pressure-diameter curves of the LES have been summarized in Fig. 3.

Splincter rclaxation. LES pressure during relaxation produced by esophageal distension was estimated in

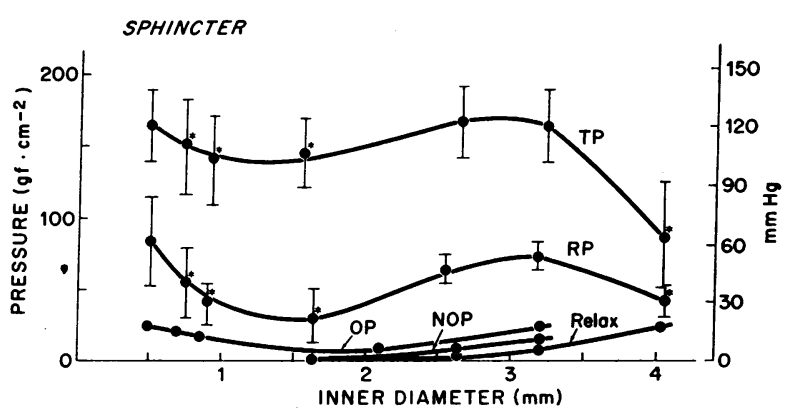

FIgURE 3 Pressure-diameter curves of the LES. (RP) is pressure-diameter curve of LES in resting state in 17 animals. (TP) is the curve of LES upon electrical stimulation in 13 animals. Pressure-diameter curve of relaxed sphincter during esophageal distension was obtained in nine animals. (OP) and (NOP) represent mean curve of the oxygenated and nonoxygenated LES preparations. (*) indicates significant $(P<0.05)$ change from the pressure at diameter of $0.51 \mathrm{~mm}$. Note that the pressure at $0.51 \mathrm{~mm}$ is similar to pressure at diameter of 2.64 and $3.20 \mathrm{~mm}$ for both resting and tetanic pressures. However, there are significant drops in pressures at intermediate diameter of $0.76,0.91$, and $1.63 \mathrm{~mm}$. At diameter of $4.06 \mathrm{~mm}$ pressure falls again. 
nine animals. No pressure was recorded for probes with diameters less than $1.63 \mathrm{~mm}$. With further increase in probe size the pressure gradually increased to $26 \pm 6 \mathrm{gf}$. $\mathrm{cm}^{-2}$ with a probe of $4.06 \mathrm{~mm}$ OD. These pressures measure the passive resistance remaining in the sphincter after active inhibition of muscular activity.

Tetanic contractions. Because the lower esophagus of the rat is composed of striated muscle in inner as well as outer layers, tetanic contraction could be produced on direct electrical stimulation. The pressure-diameter curves of the tetanized LES obtained in 13 animals were qualitatively similar to those of the resting LES, although the pressures were much higher (Fig. 3).

\section{Pressure-diameter curves of the isolated LES}

The pressure-diameter curves of the viable, oxygenated LES at rest in 10 preparations were similar to the resting LES in the intact animals but the pressures were much lower. No pressure was recorded in the 10 nonoxygenated preparations at diameters smaller than 1.6 $\mathrm{mm}$. The pressure rose almost linearly with increasing diameters. This behavior was similar to that of the relaxed LES in intact animals (Fig. 3).

Pressure-diameter relationship of the esophagus in intact animals

The mean pressure in the resting abdominal esophagus in 10 animals was $5.0 \pm 0.1, \mathrm{SD} \mathrm{gf} \cdot \mathrm{cm}^{-2}$ at a diameter of $0.51 \mathrm{~mm}$ and this increased to $53 \pm 20 \mathrm{gf} \cdot \mathrm{cm}^{-2}$ at a diameter of $4.06 \mathrm{~mm}$ (Fig. 4). Esophageal distension provoked peristaltic contraction in the esophagus (secondary peristalsis). The peak pressure of the peristaltic contraction was $20 \pm 6 \mathrm{gf} \cdot \mathrm{cm}^{-2}$ at a diameter of $0.51 \mathrm{~mm}$

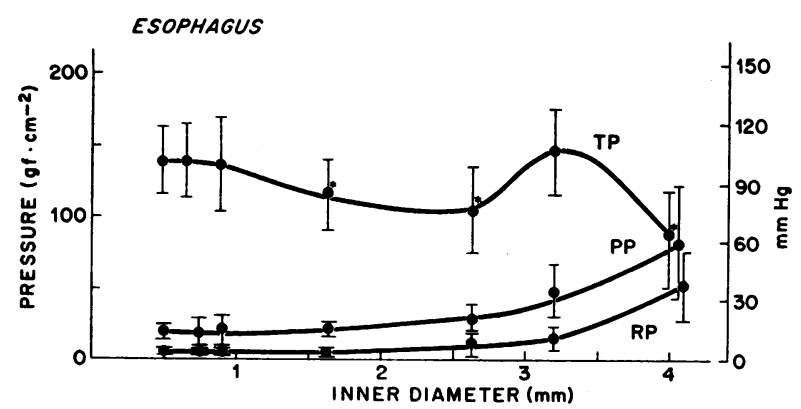

FIgURE 4 Pressure-diameter curves of the body of the esophagus. (RP) is pressure-diameter curve of the resting esophagus. (PP) is the curve of the peak pressure of the peristaltic wave which was induced by upper esophageal distension with water. (TP) is the curve of tetanically contracted esophagus produced by electrical stimulation. Each point is a mean value in 10 animals $\pm S D$. Points indicated by $\left(^{*}\right)$ are significantly different $(P<0.05)$ from the initial value on the curve at $0.51 \mathrm{~mm}$.
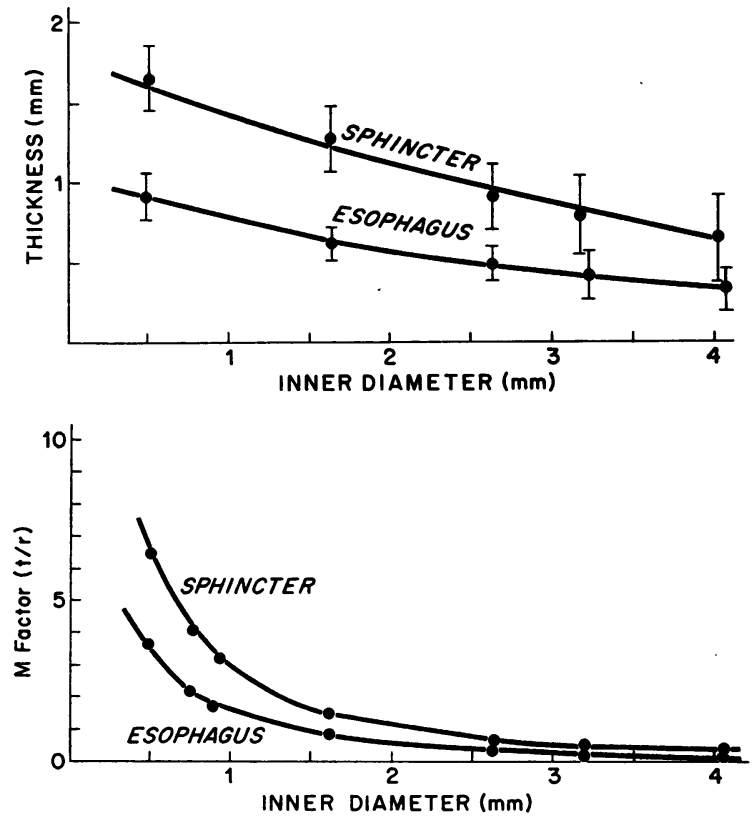

Figure 5 (Top) LES and esophageal wall thickness at different diameters. Each point is a mean value of 10 studies and bar height indicates \pm SD. Note that the wall of LES is thicker than the wall of the esophagus. (Bottom) Magnification factor of LES and esophageal wall. Mean values of the thickness at various diameters (shown on top) were divided by the respective radii to obtain $M$ factor at various luminal diameters. Note a steep increase in $\mathbf{M}$ factor at small luminal diameters.

and increased to $81 \pm 38 \mathrm{gf} \cdot \mathrm{cm}^{-2}$ at a diameter of 4.06 $\mathrm{mm}$. It is quite likely that the peak pressures with very slow infusion used in probes of small diameter may have underestimated the pressure of the peristaltic peak.

Upon tetanic contraction of the esophagus produced by electrical stimulation, the mean pressure in 10 animals at probe diameter of $0.5 \mathrm{~mm}$ was $138 \pm 26$ and $148 \pm 30$ $\mathrm{gf} \cdot \mathrm{cm}^{-2}$ at a diameter of $3.2 \mathrm{~mm}$. This difference was not statistically significant $(>0.5)$. The entire pressurediameter curve shown in Fig. 4 was qualitatively similar to that of electrically stimulated LES.

\section{Wall thickness and the magnification factor}

Thickness of the LES and the esophagus was measured at different inside diameters in 10 rats. The mean $\pm \mathrm{SD}$ of the observed values of wall thickness are shown in Fig. 5. The mean value of the wall thickness at each diameter was divided by its radius to obtain the magnification factor. The magnification factor at various diameters is shown in Fig. 5 (bottom). With small diameters there was a steep increase in $M$ factor. The mean value of pressure was divided by the $M$ factor to obtain the average wall tension. 
The tension-diameter curves of the LES and the body of the esophagus

Fig. 6 summarizes the mean tension-diameter curves of the LES at rest, upon esophageal distension, and upon electrical stimulation. The figure shows that the diameter of optimal tension development (OTD) is $3.2 \mathrm{~mm}$ which is far from closure diameter. This is true for both the resting state as well as electrical stimulation. As the diameter decreases, the tension also gradually decreases but does not cease even at the smallest diameter of 0.5 $\mathrm{mm}$ ( $15 \%$ of diameter of OTD). The optimal tension in the LES at rest is about half of that seen with electrical stimulation; the mean OTD on electrical stimulation was 312 and $147 \mathrm{gf} \cdot \mathrm{cm}^{-2}$ at rest. The mean tension in resting sphincter at $0.5 \mathrm{~mm}$ was only $13 \mathrm{gf} \cdot \mathrm{cm}^{-2}(11 \%$ of OTD at rest).

Fig. 7 shows tension-diameter curves for the esophagus at rest, during peristaltic contraction, and upon electrical stimulation. The curves for the resting esophagus are similar to relaxed LES. The mean optimal tension of the electrically stimulated esophagus was $619 \mathrm{gf} \cdot \mathrm{cm}^{-2}$; this is almost twice the optimal tension developed in the LES at rest.

\section{DISCUSSION}

The technique used in this study provides a suitable method of determining the tension-diameter curves of the muscle of the LES and the body of the esophagus in vivo. The tension developed in the sphincter muscle at rest increased with increasing diameter, reached a maximum, and then decreased with further increase in diameter. The diameter at which maximal tension is de-

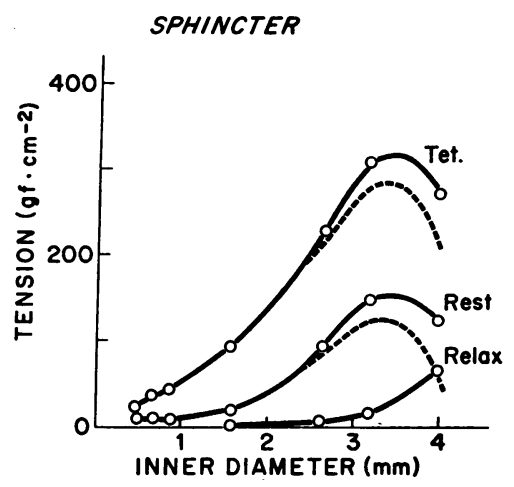

FIgURE 6 Tension-diameter curves of LES. Each point is calculated from the mean value of pressure and the $M$ factor. Tension-diameter curves of tetanized sphincter (Tet.), sphincter at rest (Rest), and of relaxed sphincter produced by esophageal distension are shown. The dotted line gives the active tension (i.e., tension at rest or on stimulation minus the passive tension of the relaxed sphincter). Note that OTD occurred around a diameter of 3.2 $\mathrm{mm}$. Note also that some tension development occurred even at a diameter of $0.51 \mathrm{~mm}$.

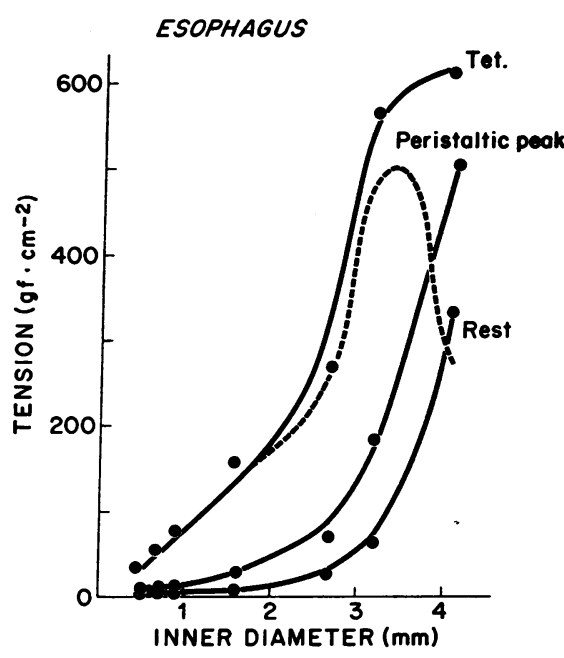

FIgURE 7 Tension-diameter curves of the esophagus during rest, during peristaltic contraction, and on tetanic stimulation (Tet.). These points were calculated from mean values of pressure and $M$ factor at various diameters. Dotted line represents the curve of active tension. Note that active OTD occurred at a diameter of about $3.2 \mathrm{~mm}$.

veloped is the diameter of OTD. These tension-diameter curves are qualitatively somewhat similar to the lengthtension curves of other muscles studied in vitro $(15,16)$. During contraction of the LES produced by electrical stimulation, much higher values of tension were obtained than in the resting state but the shape of the tension-diameter curve did not change; the optimal tension was again developed around a diameter of $3.2 \mathrm{~mm}$. In the intact animal at rest, the tension developed was almost half of what was produced on electrical stimulation. This indicates that at rest the sphincter muscle was active well below its capacity.

Studies have been performed on strips of circular smooth muscle of the LES of opossum by Lipshutz and Cohen (17). They (17) designated the length just before development of any passive tension as the "initial" length; they then applied incremental increase in length and determined the length of OTD in response to chemical stimulation with acetylcholine. The OTD occurred at a length about $25 \%$ above the "initial" length. This response is similar to that of trachealis smooth muscle which starts to show passive tension at a length about $80 \%$ of the length of OTD (18). Lipshutz and Cohen's study could not define the shortest length at which active tension could be developed. In trachealis muscle active tension ceases to develop at a length $10 \%$ of the length of OTD. The results of such in vitro studies may not be directly compared with our in vivo study. Our studies show that in the LES actively inhibited by esophageal distension, tension first appears at a diameter of $1.6 \mathrm{~mm}$ which is about $50 \%$ of the diameter of OTD. The OTD 
occurred at $3.2 \mathrm{~mm}$-a diameter much larger than the resting (closure) diameter of the LES.

Some active tension ( $11 \%$ of optimal tension) was present in the sphincter muscle when its diameter was $15 \%$ of the diameter of OTD; it was not possible to test smaller diameters. In contrast, in the voluntary muscle and cardiac muscle, the active tension falls to zero at a length of 40 and $35 \%$ of the length of OTD respectively (19). We suggest that the ability to develop tension at very small diameters may characterize the behavior of sphincter muscle that acts to keep the lumen closed. When the ability to develop tensions ceases at larger lengths, such as happens in tracheal smooth muscle, luminal occlusion cannot take place (18).

The wide diameter of OTD and the small diameter, at which active tension can be developed in the sphincter muscle, make the sphincter very versatile. As the sphincter lumen decreases and approaches closure, a small tension is enough to produce adequate intraluminal pressure because of the mechanical advantage accorded by the steeply increasing wall thickness/radius ratio or the $\mathrm{M}$ factor. This property of active tension development even at very small diameters, ensures sphincter closure. As the sphincter lumen is dilated, the mechanical advantage as measured by the $\mathrm{M}$ factor gradually decreases. The sphincter miscle can now develop more tension as it approaches the diameter of the OTD. This ensures adequate intraluminal pressure at large diameters. Thus, the mechanical factors of the sphincter and its tension diameter relationship interact in such a way that adequate intraluminal sphincter pressures can develop at widely varying diameters. Nevertheless, there are some intermediate diameters when the LES pressure is significantly lower than that near closure or at the diameter of OTD. This would suggest that the use of manometric catheters of different size may modify the result of LES pressure obtained by different investigators using widely varying sizes of catheter assembly.

\section{ACKNOWLEDGMENTS}

We wish to thank Doctors H. J. Binder, H. D. McIntosh, and G. E. Moellmann for helpful suggestions.

This study was supported by the Rose Brothers Fund at Yale University, the American Machine and Foundry Foundation, the U. S. Public Health Service Research grant R01 AM 16021-01, and a grant from Southwestern Medical Foundation, Dallas, Tex.

\section{REFERENCES}

1. Ingelfinger, F. J. 1958. Esophageal motility. Physiol. Rev. 38: 533 .

2. Code, C. F., and J. F. Schlegel. 1968. Motor action of the esophagus and its sphincters. Handb. Physiol. 4 (Sect. 6, Chapter 90) : 1821.

3. Burton, A. C. 1951. Physical equilibrium of the small blood vessels. Am. J. Physiol. 164: 319.

4. Burton, A. C. 1962. Physical principles of circulatory phenomena: the physical equilibria of the heart and blood vessels. Handb. Physiol. 1(Sect. 2, Chapter 6) : 85.

5. Botha, G. S. M. 1962. The gastro-esophageal junction. Little, Brown and Company. 68, 157.

6. Winans, C. S., and L. D. Harris. 1967. Quantitation of lower esophageal sphincter competence. Gastroenterology. $52: 773$.

7. Rinaldo, J. A., Jr., and J. F. Levey. 1968. Correlation of several methods for recording esophageal sphincter pressures. Am. J. Dig. Dis. 13: 882.

8. Pope, C. E., II. 1970. Effect of infusion on force of closure measurements in human esophagus. Gastroenterology. 58: 616 .

9. Hollis, J. B., and D. O. Castell. 1972. Amplitude of esophageal peristalsis as determined by rapid infusion. Gastroenterology. 63 : 417.

10. Zabinski, M. P., P. Biancani, A. Phillips, and H. M. Spiro. 1972. On the accuracy of pressure measurements in esophageal manometry. Gastroenterology. 62: 833 . (Abstr.)

11. Lund, G. F., and J. Christensen. 1969. Electrical stimulation of esophageal smooth muscle and effects of antagonists. Am. J. Physiol. $217: 1369$.

12. Tuch, A., and S. Cohen. 1973. Lower esophageal sphincter relaxation: studies on the neurogenic inhibitory mechanism. J. Clin. Invest. 52: 14.

13. Goyal, R. K., P. Biancani, A. Phillips, and H. M. Spiro. 1971. Mechanical properties of the esophageal wall. $J$. Clin. Invest. 50: 1456 .

14. Diem, K. 1968. Documenta Giegy Scientific Tables. Giegy Pharmaceuticals, Ardsley, N. Y. 6th edition. 215.

15. Wilkie, D. R. 1956. Mechanical properties of muscle. Br. Med. Bull. $12: 177$.

16. Zeirler, K. L. 1968. Mechanics of muscle contraction and its energetics. In Medical Physiology. V. B. Mountcastle, editor. The C. V. Mosby Co., St. Louis. 12th edition. 2 : 1128.

17. Lipshutz, W. H., and S. Cohen. 1971. Physiological determinants of lower esophageal sphincter function. Gastroenterology. 61: 16

18. Stephens, N. L., E. Kroeger, and A. J. Mehta. 1969. Force-velocity characteristics of respiratory airway smooth muscle. J. Appl. Physiol. 26: 685.

19. Sonnenblick, E. H. 1962. Implications of muscle mechanics in the heart. Fed. Proc. 21: 975. 